\title{
Fracture precursors in disordered systems
}

\author{
G. Meacci ${ }^{1,2}$, A. Politi ${ }^{1}$, and M. Zei ${ }^{3}$ \\ 1 Istituto Nazionale di Ottica Applicata, L.go E. Fermi 6, 50125 Firenze, Italy \\ ${ }^{2}$ Max Planck Institute for Physics of Complex Systems, Nöthnitzer Straße 38, 01187 Dresden, Germany \\ 3 CECM-CNRS-Vitry, 15 rue Georges Urbain, 94407 Vitry-sur-Seine, France
}

(Dated: November 7, 2018)

\begin{abstract}
A two-dimensional lattice model with bond disorder is used to investigate the fracture behaviour under stress-controlled conditions. Although the cumulative energy of precursors does not diverge at the critical point, its derivative with respect to the control parameter (reduced stress) exhibits a singular behaviour. Our results are nevertheless compatible with previous experimental findings, if one restricts the comparison to the (limited) range accessible in the experiment. A power-law avalanche distribution is also found with an exponent close to the experimental values.
\end{abstract}

PACS numbers: $\quad 46.50 .+\mathrm{a}, 62.20 . \mathrm{Mk}, 05.70 . \mathrm{Ln}$

Fractures are very complex phenomena which involve a wide range of spatial and sometimes temporal scales. Accordingly, the development of a general theory is quite an ambitious goal, since it is not even clear whether a continuous coarse-grained description makes sense; additionally, for the very same reason, realistic simulations are almost unfeasible. However, such difficulties have not prevented making progress on several aspects of fracture dynamics such as propagation velocity, roughness, or the failure time under a constant stress [1, 2, 3]. In this paper we are interested in studying the development of the socalled precursors, microcracks preceding the macroscopic fracture in a brittle disordered environment. Some recent experiments [4, 5, 6] suggest that we are in the presence of a critical phenomenon, although the accuracy is not yet high enough not only to discuss its universality properties, but also to assess the order of the transition.

Fractures are typically studied either by increasing the strain or the stress and recording the acoustic emissions generated by the microfractures preceding the final breakup. In both cases, there is experimental evidence that the probability density $N(\varepsilon)$ of microfractures with energy between $\varepsilon$ and $\varepsilon+d \varepsilon$, follows a power law

$$
N(\varepsilon) \sim \varepsilon^{-\beta}
$$

although it has been found that different materials are characterized by different values of the exponent $\beta: 1.3$ in synthetic plaster 7], 1.51 in wood [4], 1.9 in fiberglass [5], and 1.25 in paper [6].

On the other hand, the cumulative energy emitted while approaching the fracture exhibits a qualitatively different behaviour depending whether the strain or the stress is controlled. In the former case, after a bond breaks, lattice rearrangements lead to a stress reduction which, in turn, increases the overall stability. As a consequence, a critical behaviour can be realistically observed only in the latter case. For instance, in Refs. [4, 5] it was found that

$$
E\left(P_{r}\right) \sim\left(\frac{P_{c}-P}{P_{c}}\right)^{-\lambda}:=P_{r}^{-\lambda}
$$

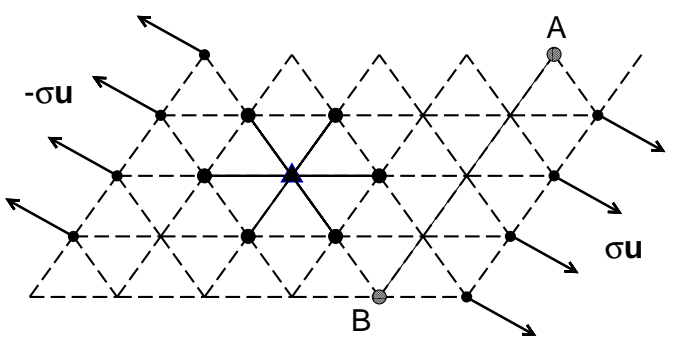

FIG. 1: Sketch of the triangular lattice. The solid lines refer to the nearest neighbour interactions involving the site denoted by a full triangle. Left and right boundaries are pulled apart by a force $\pm \sigma \mathbf{u}$. Periodic boundary conditions are assumed along upper and lower borders (e.g., the point $A$ can be identified with $B$ ).

where $E$ is the cumulative energy released up to pressure $P, P_{c}$ is the critical pressure corresponding to the macroscopic failure, and $P_{r}$ is the so-called reduced parameter. In the past, simplified models have been introduced in the hope to capture the essential ingredients of the process. Among them, random fuse networks have been quite popular, since only one scalar variable (the electric current) is needed to describe the onset of a macroscopic failure 8 . After the concept of self-organized criticality (SOC) was introduced by P. Bak [9], the possible interpretation of fractures as instances of such critical phenomena became an appealing perspective to many researchers. As a result, probabilitstic models inspired by the simplest SOC ideas have been introduced and numerically investigated [10]. Altogether, it has emerged a scenario of the fracture as a critical phenomenon, but the relative "distance" between models and physical reality leaves doubts about the validity of such conclusions. 
More realistic models where each particle feels the force field induced by its nearest neighbours were already introduced in the '80s 11, 12, 13, 14], when preliminary studies of small 2-dimensional lattices with quenched disorder have been carried out. However, only in the late '90s it has become possible to simulate sufficiently large systems to attempt a scaling analysis. In Ref. [15] a square lattice with two- and three-body interactions was studied, finding $\beta=2.5$ in agreement with the mean-field behaviour of the fiber bundle model [16, 17], but larger than the experimental values. On the other hand, large statistical uncertainties prevent drawing any conclusion about the scaling behaviour of $E\left(P_{r}\right)$.

In this Letter, studying a slightly simpler model [18], we are able to investigate the behaviour of $E\left(P_{r}\right)$ obtaining results that are compatible with the Lyon experiment 4]. However, having here investigated a wider range of parameter values, we are led to exclude a divergence of $E\left(P_{r}\right)$ near the critical point.

Our model consists of point-like particles sitting on a $2 \mathrm{~d}$ triangular lattice with nearest-neigbour interactions mediated by central forces (see solid lines in Fig 1). More precisely, the force $\mathbf{f}_{i j}$ acting on the $i$ th particle due to the interaction with the $j$ th one is

$$
\mathbf{f}_{i j}=F_{i j}\left(\left|\mathbf{r}_{i}-\mathbf{r}_{j}\right|\right) \frac{\mathbf{r}_{i}-\mathbf{r}_{j}}{\left|\mathbf{r}_{i}-\mathbf{r}_{j}\right|}
$$

where $\mathbf{r}_{i}$ denotes the $i$ th particle position, $|\cdot|$ represents the modulus operation. Moreover, $F_{i j}(x=a)=$ 0 , with $a$ being the lattice spacing at equilibrium and $k_{i j}=-d F_{i j} /\left.d x\right|_{a}>0$ so as to ensure stability. Moreover, as soon as the mutual distance $\left|\mathbf{r}_{i}-\mathbf{r}_{j}\right|$ becomes larger than some threshold $a_{i j}^{*}$, the interaction strength is irreversibly set equal to 0 . As the $a_{i j}^{*}$ 's are generally chosen close to $a$ (see also Ref. 12]), only small deviations from equilibrium can be expected and, accordingly, the force can be linearized. Here, we have however preferred the equivalent choice (at the first order level) $F(x)=-\left(x^{2}-a^{2}\right) /(2 a)$, since it avoids the computation of a square root for each bond, thus ensuring faster simulations.

Furthermore, an external force $\sigma \mathbf{u}(-\sigma \mathbf{u})$ is applied to each particle of the right (left) boundary along a direction $\mathbf{u}$ orthogonal to the edges, while periodic boundary conditions are assumed along the upper and lower boundaries. Since we are interested in investigating the fracture process in a nearly static regime, a damping term $-\gamma \dot{\mathbf{r}}_{i}$ has been added to the forces acting on the $i$ th particle. The last key element of the model is disorder, which can be introduced by assuming that either the bond strengths $k_{i j}$ or the thresholds $a_{i j}^{*}$ are distributed according to some law. Here, we assume $a_{i j}^{*}=a^{*}$ and a dichotomic distribution of bond strengths: a fraction $c$ of them is set equal to 0 from the very beginning, while the rest are set, without loss of generality, equal to 1. As pointed out in Ref. [13], this choice is equivalent to a dichotomic distribution of

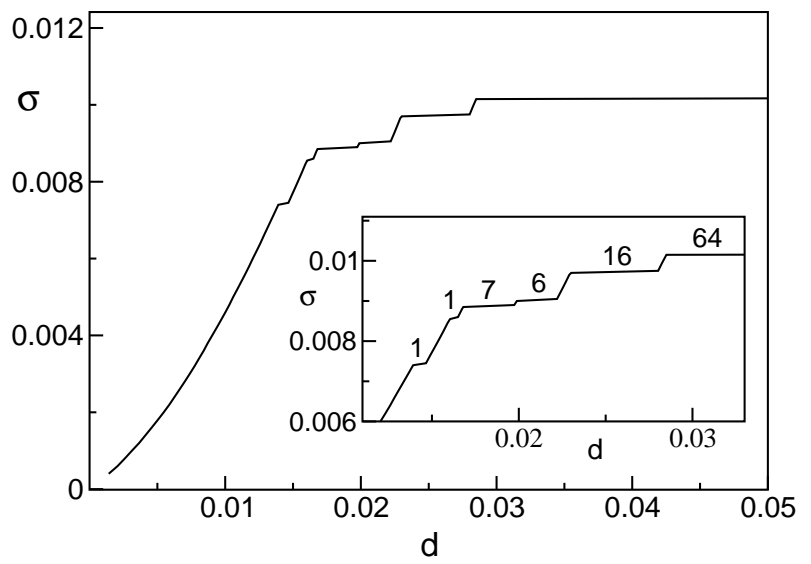

FIG. 2: The stress $\sigma$ as a function of the strain $d$, for one disorder realization in a lattice with $40 \times 42$ particles. Part of the curve is zoomed in the inset so as to identify single avalanches (the numbers over the horizontal steps represent the number of broken bonds in each avalanche).

thresholds.

It is convenient to express all variables in adimensional units, since this helps scaling out some parameters. In particular, the lattice spacing $a$ and the particle masses can be both set equal to 1 . This implies that the modulus $\sigma$ of the applied force can be identified with the stress. A parameter that cannot be scaled out is the threshold, here fixed equal to $a^{*}=1.04$. This choice, besides being compatible with the linearization of the force field, allows us reaching sizeable large-scale deviations from a purely crystalline structure (we have been able to study lattice sizes up to $L=80$ ).

The equations of motion have been integrated by using a Runge-Kutta algorithm with $\gamma=0.7$ and a time step equal to 0.3. We have verified that this choice guarantees the fastest convergence (in CPU-time units) to the asymptotic state[19]. Finally, we have chosen to fix the fraction of initially missing bonds equal to $c=0.3$, a value close to, but definitely below, the rigiditypercolation threshold $\left(c_{r}=0.3398\right)$.

Numerical experiments consist in monitoring several observables while the stress is increased until a macroscopic failure occurs at $\sigma=\sigma_{c}$. In order to ensure that the sampled configurations remain close to equilibrium during the whole stretching process, (i) $\sigma$ is slowly increased, (ii) additional relaxation loops are allowed when new bonds break. One of the relevant observables is the strain $d=\overline{\left|\left(\mathbf{r}_{i}-\mathbf{r}_{i}^{0}\right) \cdot \mathbf{u}\right|}$, where the overline denotes the average over all particles of the left and right edges and $\mathbf{r}_{i}^{0}$ represents the equilibrium position. As a typical example of the observed phenomenology, in Fig. 2] we plot the stress-strain curve for one realization of the disorder. After an initially monotonous growth, a series of steps follows, each corresponding to the "simultaneous" breaking of $s$ bonds. Since the same amount of energy is 


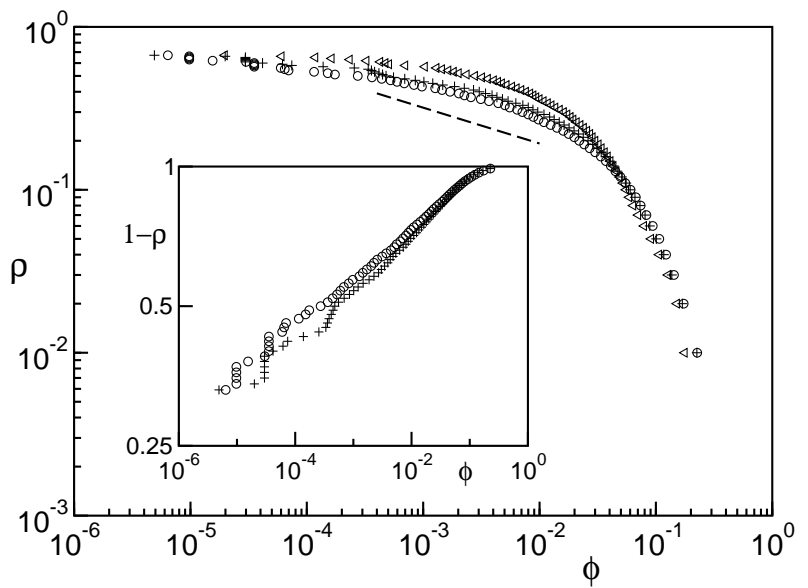

FIG. 3: Average cumulative fraction of broken bonds $\rho$ versus the reduced stress $\phi$ for $L=20$ (triangles), 40 (plusses), and 80 (circles). The dashed curve corresponds to the scaling behaviour observed in Ref. [4]. In the inset, $1-\rho$ is plotted versus $\phi$ with the same meaning of the symbols.

released in each bond breaking, it is natural to identify $s$ with $\varepsilon$ and interpret the phenomenon as the occurrence of an avalanche.

Another relevant observable is the number $N(\sigma)$ of bonds broken at stresses smaller or equal to $\sigma$, a quantity analogous to the cumulative energy $E$ in Eq. (2). Moreover, having the possibility to average over many different realizations of the disorder (actually, we have studied 1000 realizations for each lattice size), it is also necessary to define a meaningful way of performing ensemble averages. We have decided to consider the fraction

$$
\rho=\frac{N(\sigma)}{N\left(\sigma_{c}\right)},
$$

as the independent variable $\left(N\left(\sigma_{c}\right)\right.$ being the total number of broken bonds in each realization) and to average the stress values where $\rho$ is attained. Moreover, analogously to Ref. [4], where the reduced pressure $P_{r}$ has been introduced, here we define the average reduced stress

$$
\phi=\left\langle\frac{\sigma_{c}-\sigma}{\sigma_{c}}\right\rangle
$$

where $\langle\cdot\rangle$ denotes an average over disorder. Our simulations indicate that, in the thermodynamic limit $L \rightarrow \infty$, the average becomes irrelevant. Indeed, we have verified that $\sigma_{c}$ is a self-averaging quantity, by observing that the normalized variance

$$
\Delta_{\sigma}=\frac{\left\langle\sigma_{c}^{2}\right\rangle-\left\langle\sigma_{c}\right\rangle^{2}}{\left\langle\sigma_{c}\right\rangle^{2}}
$$

decreases as $\Delta_{\sigma} \simeq L^{-2 / 3}$ with increasing $L$.

The resulting behavior for $L=20,40$, and 80 is plotted in Fig. 3. The data for the $L=40$ and 80 nicely

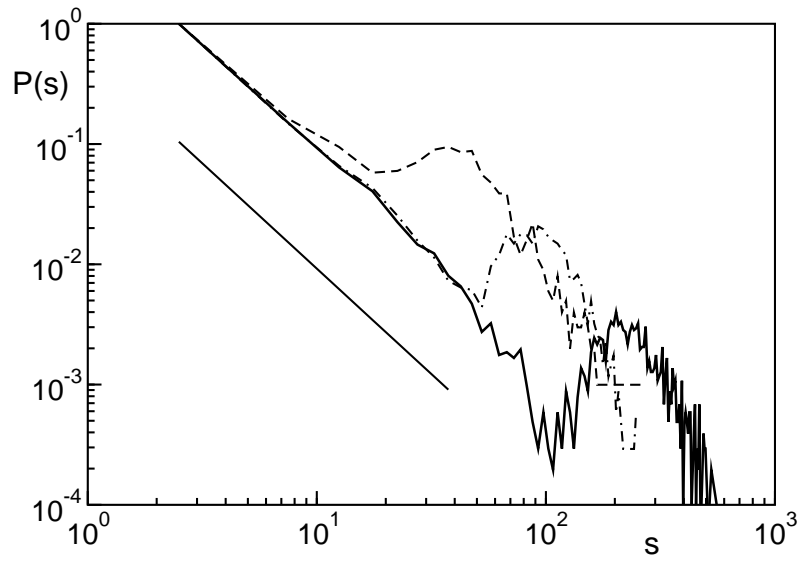

FIG. 4: Probability density $P(s)$ of avalanches preceding the macroscopic failure, scaled to its maximum value. Dashed, dashed-dotted, and solid lines refer to $L=20,40$, and 80, respectively. The straight line, corresponding to a decay $s^{-1.75}$ is the fit - shifted for clarity - of data for $L=80$

overlap, indicating that finite-size effects are already negligible for $L \approx 40$. In comparison with the experiment of Ref. 4], the reduced stress here covers a three-times wider range; this allows us ruling out the existence of a power-law behaviour of $\rho$. On the other hand, if we restrict the analysis to the range accessible in the experiment, we do not find relevant differences with respect to the experiment itself, as indicated by the dashed line in Fig. 3. Next, one notices that $\rho$ remains strictly smaller than 1 even close to $\phi=0$, suggesting that the fraction of bonds broken in the macroscopic failure is finite. However, the most refined presentation in the inset reveals that, though slowly, $\rho$ eventually converges to 1 . Indeed, by fitting the small- $\phi$ region with $\rho=\rho_{0}-b \phi^{\eta}$, we find that $\rho_{0}$ increases with $L$ and is equal already to 0.94 for $L=80$. As for the $\eta$ value, our best estimate is $\eta=0.12 \pm 0.01$. Although the fit is quite good, the smallness of $\eta$ leaves doubts about the effective behaviour of $\rho$ in the vicinity of the macroscopic failure.

The dependence of $\rho$ on $\phi$ sheds light on the critical behaviour around the onset of the macroscpic fracture. It is interesting to investigate also the dependence of the total number of broken bonds $N\left(\sigma_{c}\right)$ on $L$. Here, we have studied too a few sizes, to convincingly determine the scaling behaviour; however, assuming that eventually $N\left(\sigma_{c}, L\right)=L^{\alpha}$, we find that $\alpha \approx 1.5$, a value that is compatible with the simulations of the same system with imposed strain 18.

Another characterististic of fracture processes often studied both in experiments and simulations is the distribution of microfractures (i.e. avalanches). In the present context, this amounts to computing the number $N_{s}$ of avalanches of size $s$; in order to increase the statistics, we sum over all disorder realizations. The probability distributions for $L=20,40$, and 80 are plotted in Fig. [4 where 


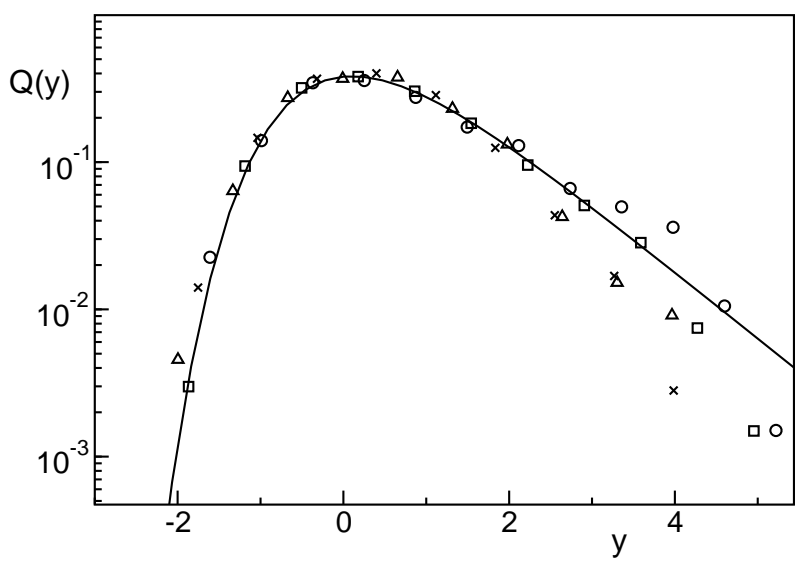

FIG. 5: The probability density $Q(y)$ of the final avalanche size $y$. The size is shifted around the mean value and scaled to ensure a unit variance. Crosses, triangles, squares and circles correspond to $L=10,20,40$, and 80 . The solid line corresponds to the Gumbel distribution.

$N_{s}$ is scaled to its maximum value $\left(P(s)=N_{s} / N_{s}^{\max }\right)$. All curves exhibit a seemingly power-law decay followed by a peak at large $s$, which is clearly a finite-size effect, since it corresponds to avalanche sizes that are comparable with the lattice size. By fitting $P(s)$ in the meaningful $s$ range with $a s^{-\beta}$, we find $\beta=1.75 \pm 0.06$, a value that lies inside the interval corresponding to the various experimental measures, [1.25, 1.9], [20]. Finally, we have investigated the size distribution of the macroscopic avalanche. The results plotted in Fig. 5 reveal increasing deviations from a Gaussian behaviour, when $L$ is increased. The Gumbel distribution, derived to describe extreme-value statistics (see the solid line) appears to provide a more convincing description of the avalanche distribution.

Once the fracture process is recognized to be a nonequilibrium phase transition, it becomes important to establish the order of the phenomenon. From the existing literature, it is unclear whether we are in front of a second order transition or the spinodal point of a first order transition [15, 21]. Such an uncertainty is mainly due to the fact that a convincing order parameter has not yet been identified. On the basis of our simulations and of the Lyon experiment, it seems reasonable to consider the cumulative fraction of broken bonds as a proper order parameter. As a consequence, since the contribution of the final avalanche is increasingly negligible in the large size limit, we are led to conclude that the fracture is indeed a continuous transition. However, it seems necessary to investigate yet larger systems in order to convingly identify the asymptotic scaling behaviour of $\rho$ in the vicinity of the critical point.

While we are quite confident about the validity of our conclusions in this specific model (since the same scenario arises when both the lattice geometry and the direction of the applied stress is changed), we should add that a different behaviour has been found for rectangular distribution of elastic constants. In such a case, although the numerical results are again compatible with the experimental ones (within the experimentally accessible range), $\rho(\phi)$ seems to exhibit a discontinuity at $\phi=0$. It thus not illogical to conjecture that the fracture may fall within (a few) different universality classes, depending on some microscopic details of the model that have still to be identifed.

This work has been partially funded by the FIRBcontract n. RBNE01CW3M_001.

[1] J. Fineberg and M. Marder, Phys. Rep. 313, 2 (1999).

[2] E. Bouchaud, J. Phys.: Condensed Matter 9, 4319 (1997).

[3] Y. Pomeau, C. R. Acad. Sci. Paris 314, 553 (1992).

[4] A. Garcimartin, A. Guarino, L. Bellon, and S. Ciliberto, Phys. Rev. Lett. 79, 3202 (1997).

[5] A. Guarino, S. Ciliberto, A. Garcimartin, M. Zei, and R. Scorretti, Eur. Phys. J. B 26, 141 (2002).

[6] L.I. Salminen, A.I. Tolvanen, and M.J. Alava, Phys. Rev. Lett. 89, 185503 (2002).

[7] A. Petri, G. Paparo, A. Vespignani, A. Alippi, and M. Costantini, Phys. Rev. Lett. 73, 3423 (1994).

[8] Statistical models for the fracture of disordered media, ed. by H.J. Herrmann and S. Roux (North-Holland, Amsterdam 1990).

[9] P. Bak, How nature works, Springer-Verlag (New-York, 1996).

[10] G. Caldarelli, F.D. Di Tolla, and A. Petri, Phys. Rev. Lett. 77, 2504 (1996).

[11] B.K. Chakrabarti, D. Chowdury, and D. Stauffer, Z. Phys. B: Condensed Matter, 62, 343 (1986).

[12] P.D. Beale and D.J. Srolovitz, Phys. Rev. B 37, 5500 (1988).

[13] A. Hansen, S. Roux, and H.J. Herrmann, J. Phys. (France) 50, 733 (1989).

[14] G.N. Hassold and D.J. Srolovitz, Phys. Rev. B 39, 9273 (1989).

[15] S. Zapperi, P. Ray, H.E. Stanley, and A. Vespignani, Phys. Rev. E, 59, 5049 (1999).

[16] P.C. Hemmer and A. Hansen, ASME J. Appl. Mech. 59, 909 (1992).

[17] M. Kloster, A. Hansen, and P.C. Hemmer, Phys. Rev. E 56, 2615 (1997).

[18] A. Politi and M. Zei, Phys. Rev. E, 63, 056017 (2001).

[19] The relaxation process becomes, however, increasingly slow, when $L$ is increased and this problem should be seriously considered, if one were interested in studying yet larger systems.

[20] A similar $\beta$-value (1.7) has been recently obtained in a strain-controlled numerical experiment: M. Minozzi, G. Caldarelli, L. Pietronero and S. Zapperi, cond-mat/0207433 (2002).

[21] D. Sornette and J.V. Andersen, Eur. Phys. J. B 1, 353 (1998). 